\author{
Visión Electrónica \\ Más que un estado sólido \\ https://doi.org/10.14483/issn.2248-4728
}

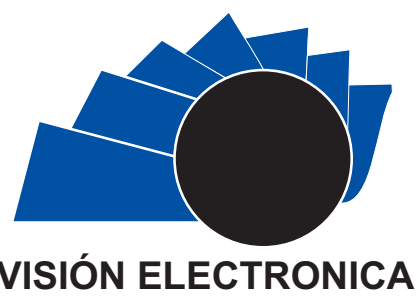

A Current Vision

\title{
State of art in quantum computing $(Q C)$ with open source platforms
}

\author{
Estado del arte en computación cuántica con plataformas de fuente abierta
}

\author{
Henry Mauricio Cárdenas-Martínez ${ }^{1}$, Jhonatan Camilo Laverde-Forero ${ }^{2}$, \\ Gerardo Castang-Montiel ${ }^{3}$
}

\section{INFORMACIÓN DEL ARTICULO}

Historia del articulo

Enviado: 04/10/2018

Recibido: 01/11/2018

Aceptado: 02/12/2018

\section{Keywords:}

Algorithms,

Bit,

Classical computing,

Overlap,

Processor,

Qubit.

\section{Palabras clave: \\ Algoritmos, \\ Bit, \\ Computación clásica, \\ Superposición, \\ Procesador, \\ Qubit.}

\begin{abstract}
The idea behind the $Q C$ paradigm lies in the information storage capacity in amplitude values that use systems based on Qubits or quantum bits -not in bits-, and in the processing of it when transformations are required to change these amplitudes in a precise and controlled way. Therefore, the description of the states in a computer evolves obeying different algorithms than the known ones: correcting errors and digitizing arbitrarily precise calculations through limited resources. The present documentary research, carried out by the Orion research group in 2018, establishes a state of the art of knowledge in $Q C$ oriented to education as a baseline for future research whose development platforms are open source kind.

A methodology based on indexes is established to categorize and subcategorize the $Q C$, as are: fundamentals and background, $Q C$ history, concept and operation, companies that implement the $Q C$ field, applications, platforms that are managed in $Q C$, architecture, and finally the programming languages. Source databases such as IEEE-Xplore, EBSCO, and websites, were used to illustrate the fundamental concepts and developments that companies have specified in applications using this new paradigm.
\end{abstract}

RESUMEN:

La idea que subyace al paradigma de la $C C$ estriba en la capacidad de almacenamiento de información en valores de amplitud que usan sistemas basados en Qubits o bits cuánticos -no en bits-, y en el procesamiento de la misma cuando se requieren transformaciones para cambiar estas amplitudes de una manera precisa y controlada. Por lo anterior, la descripción de los estados en una computadora evoluciona obedeciendo a algoritmos distintos a los conocidos: corrigiendo errores y digitalizando cálculos arbitrariamente precisos a través de recursos limitados. La presente investigación documental, adelantada por el grupo de investigación Orión en 2018, establece un estado del arte del conocimiento en $C$ Corientada a educación como línea de base para investigaciones cuyas plataformas de desarrollo sean tipo open source. Para lo anterior, se establece una metodología basada en índices para categorizar y subcategorizar la $C C$, como lo son: fundamentos y antecedentes, historia de la $C C$, concepto y funcionamiento, empresas que implementan el campo de la CC, aplicaciones, plataformas que se manejan en la $C C$, arquitectura, y por último los lenguajes de programación. Las bases de datos fuente usadas fueron: IEEEXplore, EBSCO, y sitios web para identificar las empresas que hacen uso del paradigma $C C$.

\footnotetext{
BSc. In Data Systematization Technologist, Universidad Distrital Francisco José de Caldas, Colombia. Current position: SPIRA S.A.S. E-mail: henry.cardenas@spira.co ORCID: https://orcid.org/0000-0002-1193-0698

${ }^{2}$ BSc. In Data Systematization Technologist, Universidad Distrital Francisco José de Caldas, Colombia. Current position: JAZZPLAT Colombia. E-mail: jhonatanlf@jazzplat.co ORCID: https://orcid.org/0000-0003-3265-4175

${ }^{3}$ BSc. In Electronic Engineering, Universidad Distrital Francisco José de Caldas, Colombia. Current position: Professor at Universidad Distrital Francisco José de Caldas, Colombia. E-mail: gerardocastang@gmail.com ORCID: https://orcid.org/0000-0001-9788-5121 


\section{Introduction}

The QC main idea is to store information in the values of complex amplitudes $2^{\mathrm{N}}$ that describe the wave function of two-tier N systems (Qubits), and process this information through the unit transformations application (quantum gates), that change these amplitudes accurately and in a controlled way. It is estimated that the $\mathrm{N}$ value needed to have a useful machine is $10^{3}$ or more; realizing that even $2^{1000} \sim 10^{300}$ is much greater than the amount of protons in the Universe, [1].

However, quantum computers work in a similar way to common computers since both depend on the Turing machine concept. The Turing machine can be abstracted as an infinitely long ribbon strip divided into squares that are read by a machine, that is: a theoretical device that could develop any mathematical problem represented by an algorithm, or what is now known as the basic idea of an operating system, [2]. The mechanism is based on the fact that in each square there is a 0 or 1 , or a blank space. Under this mechanism, where the information basic unit, the bit, is represented as 0 or 1 , a read and write header is interpreted. But for the quantum case, this means that in any cell may be a 0 , 1 or both 0 and 1 , and each intermediate point.

On the other hand, today, virtual environments through educational software can generate a simulation of a specific topic where the user can interact, contributing to cognitive development and enhancement of new ways of thinking, [3]. From this point of view, video games in quantum computing field have contributed to the knowledge construction and formation of people who study the subject, because when they play, it is necessary to use and activate stimuli related to the two brain hemispheres, Interactively and mutually reinforced. Emotion, instinct, fantasy and disorder offer the opportunity to reason, deduce, analyze and synthesize rigorously.

This paper is structured, therefore, to describe the documentary investigation results that led to establish a state of the art in QC and its open access applications, emphasizing the applications made within the framework of the $\mathrm{QC}$, and what organizations and their evolution have been highlighted in this field.

\section{Methodology}

The central theme categorization was defined as follows: quantum computing fundamentals and historical background; concepts and operation of the $Q C$; free access to quantum computer platforms that were built in North America and Europe between the years 2016 and 2018; general platform architecture; quantum software programming languages -Quipper, ScaffCC / Scaffold, QWire-, among others; business sectors that work with the $Q C$ and finally $Q C$ tendencies, perspectives and challenges. The Method by indexes was used for the review construction, [4]. Figure 1 establishes the mentioned categories and the subcategories. The endorsement of this methodology was made by the Orion Research Group of the Francisco José de Caldas District University expert analysis.

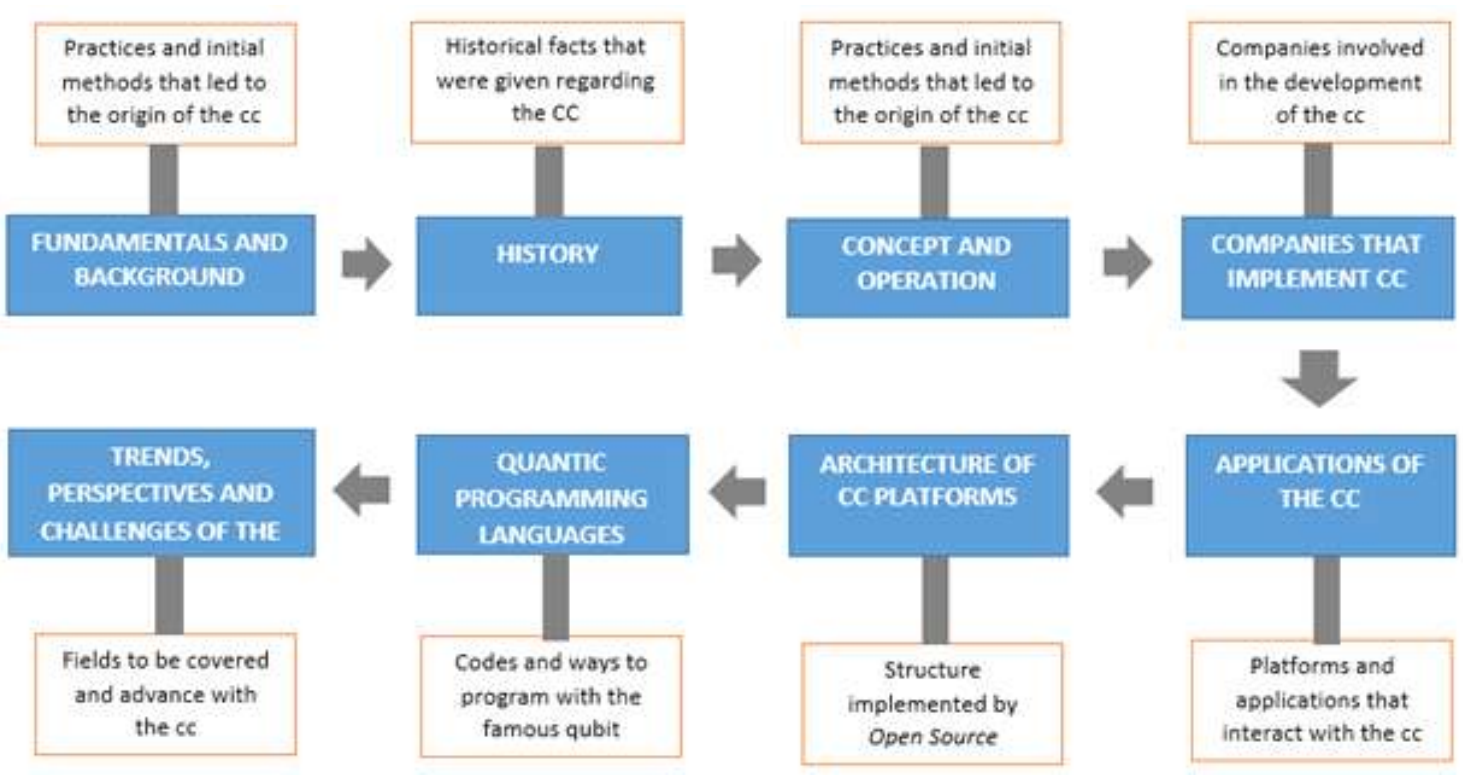

Figure 1. Implemented methodology. Source: own. 


\section{Theme development}

\subsection{Fundamentals and background}

Quantum thought had been structured through paradoxes arising in the mathematical logic field -as in the case of David Hilbert in his famous hypothesis of 1900-, which remained one of the great mathematical problems for more than half century until it was finally "solved". Cantor himself believed that there were no intermediate infinities, and called this conjecture the Continuum Hypothesis. Cantor was extremely frustrated with himself for not being able to prove it, [5] But, why is there a desire to investigate other laws and different theories about the continuum instead of those given by classical tradition?: By the discovery that Newton's and Maxwell's laws are a more general theoretical approach to this reality emanating from quantum physics.

The classical approach to quantum mechanics holds very well on the macroscopic scale of objects such as planets, airplanes, soccer balls or even molecules.

But in the "quantum scale" of atoms, electrons and individual photons, the classical approach becomes imprecise, so the theory of quantum physics must be taken into account, [6].

However, in the field of $Q C$ not everything is usually an advantage. This new technology faces challenges and difficulties that have not yet been solved. In fact, the main problems that limit $Q C$ are overheating due to the amount of operations and processes used in the code, and the problem of error tolerance, [7], [8]. This becomes critical because is needed to iterate an exponential number of times the factorization of a number of $n$ bits. This leads to an exponential expenditure of energy, given the thermodynamic model $k T$ of minimum energy per binary operation. Thus Qubits are vulnerable to errors, so a quantum error correction code (QECC) is needed to build a reliable quantum computer.

For all these reasons, the development of $Q C$ has been going on since before the current prototypes were implemented, approximately since 1900 there has been a theoretical model of quantum computing, but for different reasons it has not yet been fully transposed to make the definitive break with the paradigms of traditional computing.

In the previous sense, when one tries to use classical mechanics and electrodynamics to explain atomic phenomena, the results to which they lead are in clear contradiction with experience, [9]. If quantum mechanics is implemented to explain the "inexplicable," quantum computing is a new field of intersection of computing, mathematics, and physics, which strives to take advantage of some aspects of quantum mechanics to expand computational horizons, [10]. However, besides the known examples, finding a quantum computing application is a challenge. Designing a good quantum algorithm is a challenging task. This does not necessarily derive from the difficulty of quantum mechanics; rather, the problem lies in expectations: a quantum algorithm must be faster, and computationally less complex than any classical algorithm known for the same purpose, [11].

\subsection{History}

Quantum computers were invented in the 1980 s by Richard Feynman and his colleagues, but have recently become capable of working as well as supercomputers, [12]. In 1994, Peter Shor developed a quantum algorithm that calculated the prime factors of a large number, much more efficiently than a classical computer, [13].

However, the first person to propose a theory was Paul Benioff -of Argonne National Laboratory- where he first applied quantum theory to computers in 1981, [14]. He did this in order to take advantage of quantum laws in the computing environment. Instead of working at the level of electrical voltages he worked at the level of quantums, [15].

Towards 1985, David Deutsch gave a solid mathematical basis to Feynman's proposal. Deutsch explained how a universal quantum computer could work and described its operation as sequences of elemental operations on Qubits, [16]. For 1993 Dan Simon -from Microsoft's research departmentsuggested a theoretical problem that demonstrated the practical advantage that a quantum computer would have over a traditional one, [17]. In 1993, Charlie Bennett - along with his colleagues from IBM's Watson Research Center in New York- demonstrated how to transmit quantum information from one point in space to another without going through intermediate space. Since then, different distance marks have been broken: the record is now at about $100 \mathrm{~km},[18]$. 
After the pioneering work of D. Deutsch quantum calculus still remained as a marginal curiosity in theory until 1994 when Peter W. Shor presented quantum algorithms for factoring integers, and for extracting discrete logarithms in polynomial time, [19].

In 1996 Lok Grover created an algorithm used in quantum computation for searching in an unordered sequence of data, and with an additional need for storage space, [20].

In 1997 the first practical experiments were initiated and the doors were opened to start implementing all those calculations and experiments that had been theoretically described until then. The first secure communication experiment using quantum cryptography was successfully performed at a distance of $23 \mathrm{~km}$. In addition, the first quantum teleportation of a photon, [21], was performed.

Later, in 1998, Isaac Chuang leaded the Berkeley group that developed the first 1-Qubit quantum computer, [19]. During that same year the first 2Qbit machine was created at the University of Berkeley, California (USA). A year later, in the IBMAlmaden laboratories, the first 3-Qbit machine capable of running Grover's first search algorithm was created, [21].

In 2000, was had the most powerful IBM quantum computer with 'only' 5 Qubits of capacity; but the objective they set themselves was to be able to build one with 50 Qubits, something that would surpass the most powerful super computers of the moment. However, in order to get all that power, they would also need to improve the protection of the Qubits. Since they are very delicate and need to operate at temperatures close to absolute zero, any change in temperature could destabilize them and produce calculation errors, [22].

This is how IBM and Stanford University managed to run Shor's algorithm for the first time on the first 7Qbit quantum computer developed in Los Alamos. In the experiment the prime factors of 15 were calculated, giving the correct result of 3 and 5 using 1018 molecules, each one with 7 atoms, [23].

The Institute of "Quantum Optics and Quantum Information" at the University of Innsbruck (Austria) announced that its scientists had created the first Qbyte, a series of 8 Qubits using ion traps, [24].
In 2006, theorists and researchers from the Institute of Quantum Computing (IQC) and the Perimeter Institute of Theoretical Physics (PI) in Waterloo, along with MIT and Cambridge, presented an operational control method in the processing of quantum information that extended to 12 Qubits: they decoded it using liquid-state nuclear magnetic resonance quantum information processors, [25].

In September 2007, two U.S. research teams, the National Institute of Standards (NIST) in Boulder and Yale University in New Haven, were able to join quantum components through superconductors. Thus the first quantum bus appears, and this device -in addition- could be used as quantum memory, retaining quantum information for a short period of time before being transferred to the next device, [26].

According to the U.S. National Science Foundation (NSF), a team of scientists managed to store for the first time a Qubit (the equivalent of a "bit" of the "classical world", but in the "quantum world") inside the nucleus of a phosphorus atom, and they were able to make the information remain intact for 1.75 seconds. This period may be expandable by error correction methods, so it was a significant advance in information storage, [27].

Leonardo DiCarlo, an Argentinean resident in Holland and professor of physics at the Delft Technological University and considered an eminence at just the age of 38, in 2009 - while finishing his studies at Yale University, USA - teamed up to create the first solid-state quantum processor. "It had been done in atomic and molecular systems, but we managed to create a mini-processor with an integrated circuit", [28].

By 2011, the world's first commercially available quantum computer using the principles of quantum mechanics instead of classical mechanics was produced and sold to defense and security aerospace company Lockheed Martin [29].

Finally, it is worth mentioning that during the last two decades, Vuckovic's laboratory has tried to develop new types of quantum computer chips. Recently, it has joined forces with other companies around the world to test three different ways of isolating electrons to interact with lasers, [30]. 


\subsection{Concept and operation}

The rules and behaviours that govern quantum computing are no longer deterministic but probabilistic systems. The new measurement trend is going to be probability and entropy. Von Neumann's entropy of an overlay of Qubits measures the distribution of probabilities, and describes the exit of the state from a pure state. For a pure state there is no uncertainty during measurement. The greater the entropy, the greater the uncertainty during measurement, [31].

In the previous sense, a vital and relevant point in quantum computing with respect to the classical approach is to consider the use of Qubits: 0,1 , and 0 \& 1 at the same time, advantage to perform multiple operations simultaneously according to the number of Qubits. Opposite case to the digital or classic computation that handles bits and can only take the values of 0 or $1,[32]$.

In classical physics $F=m a($ Force $=$ mass $*$ acceleration $)$ but for quantum physics this presents an indefinition with the passage of time, so this is not ensured in a particle: the real state of the particle cannot be seen as the system itself. This has to be determined by the probability that a particle is in one state or another. The quantum computation model speaks of superposition: having more than one position at a given moment based on probability, [33].

Quantum information is in a sense a combination of two pieces of classical information: information about two physical observables that are complementary in the sense exposed by Bohr and exemplified by the duality of particles in the double slit or tunneling experiment, Figure 2, [34].



Figure 2. Tunnel effect. The upper part of the figure represents the situation described by classical physics. The lower part of the figure represents the situation described by quantum physics, [35].

\subsection{Companies that implement quantum computing}

At present, QC has progressively moved towards a complex study that has contraindications. Technology has advanced quickly in companies such as IBM [36], Google, Microsoft Corp., Canada's D-Wave Systems Inc. [37].

The later one is the first in the world to sell quantum computers. Other quantum computing studies, including the University of Maryland spin-offionQ, are developing a quantum computer of captured ions; and PsiCorp, founded by researchers from the University of Bristol, is developing a photonic quantum computer using silicon chips, [38].

And the startup Rigetti Computing [39], based on California, are striving to create machines that companies can use, [40].

For example, in the case of IBM its objective is to build quantum computers of practical applicability in the world of science and business, [41]; google with Dubbed Bristlecone, which is a system of 72 Qubits [42], wants to reach quantum supremacy; Microsoft, meanwhile, has implemented algorithms that simulate behaviors of a quantum computer such as the LIQUI software [43] and the 2000 Qubits of D-Wave -the DWave $2 \mathrm{x}$ is considered one of the most advanced and sophisticated computers today-, [44].

All these companies have advanced in their research and are competing with each other to have the supremacy of the market, by this competitive way in a few years there will be witnessed a personal quantum computer as well. Even the IEEE [45] is trying to standardize the new technologies and protocols that will be implemented with the QC, [46].

\subsection{Applications of quantum computing}

The following is a description of the main free access platforms on quantum computing in which the user can enter and interact for free. The idea behind this section was generated by a research carried out at the District University Francisco José de Caldas in the Faculty of Technology in the Telematics Engineering curricular project where it is evident that with the Quantum Game tool -free access- it was possible to complement and help promote the knowledge of what quantum computing is; with the results it could be deduced that, effectively, students relate this branch of physics and technology in a better way with the help of complementary applications. 


\subsubsection{First quantum game CAT-BOX SCISSORS}

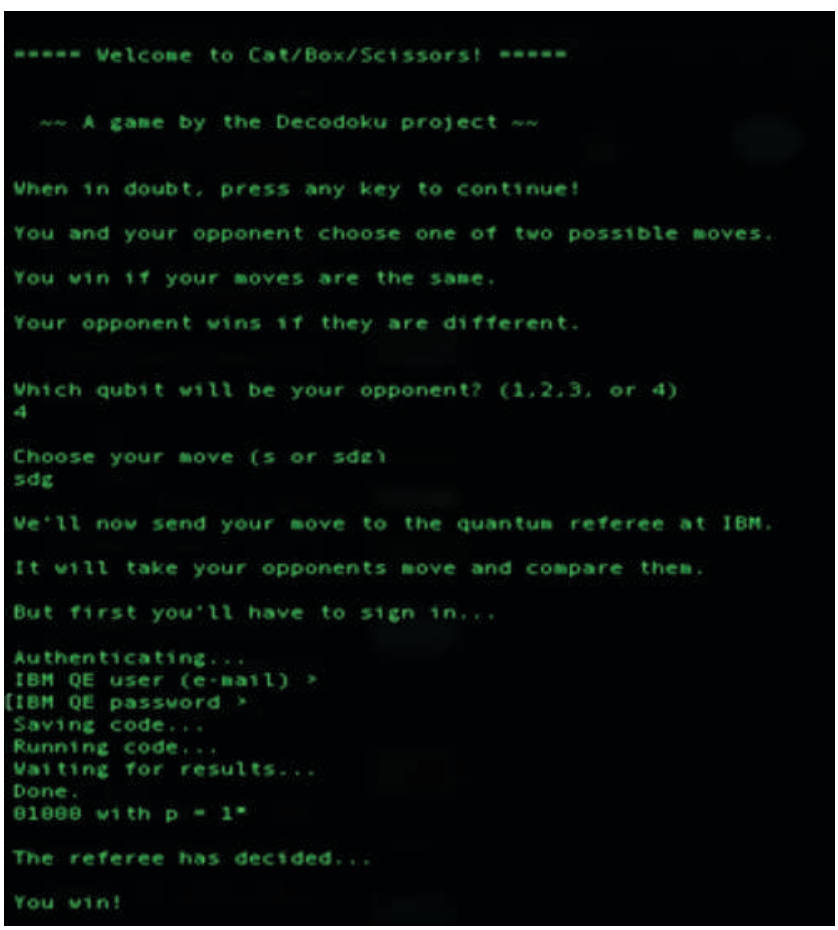

Figure 3. Quantum first game interface "Cat / box / scissors". Source: own.

It's a quantum version of stone / paper / scissors. It has five quantum bits. One of them will act as a referee and tell who wins. The other four are all potential opponents. Although only one is played at a time. For inspiration, what is possible for normal bits is that the simplest is a NOT gate. It changes bits from 0 to 1 and from 1 to 0 . Quantum computers can do this, but they can also be a bit more. For example: they can do NOT half, leaving the quantum bit in a strange limb state between $O$ and 1 . A quantum superposition, like Schrödinger's cat.

The game is available in two versions: Project $Q$ version (using the .py file); and can be played directly using the IBM Quantum Experience interface (using the .qasm file). In either case, you will need a Quantum Experience account to access the device, [47].

\subsubsection{D Puzzles}

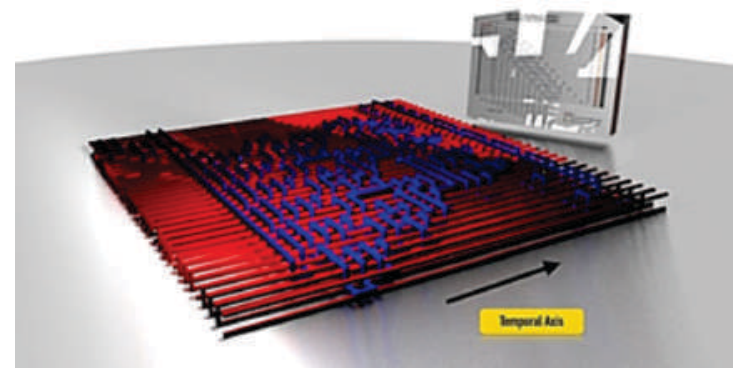

Figure 4. Quantum game interface "3D Puzzles". Source: own.

Riken Institute researcher in Saitama, Japan, Simon Devitt, developed an online game that could play a decisive role in the future of quantum programming. Not only can the game help humans create better programs, it could also help a new generation of artificial intelligence machines take on that task: a powerful way to visualize quantum programs as $3 \mathrm{D}$ grids with interlaced sections representing how information is stored and processed. To optimize them, it is necessary to simplify the grid by moving, shrinking, stretching and reconfiguring the interlaced sections so that they preserve the same topology, [48].

\subsubsection{Quantumawesomeness}

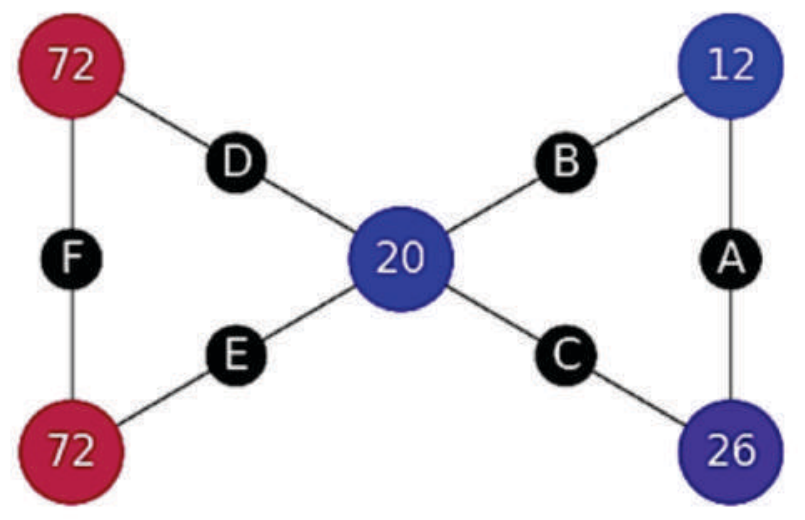

Figure 5. Quantum game interface "Quantum awesomeness". Source: own.

Quantum Awesomeness is a simple puzzle game. Each puzzle consists of a grid of colored dots with numbers at each point. Each point must have a color and number similar to one of its neighbors. The player's job is to look for these similarities and use them to match all the points. There will be a Game Over condition when the puzzles become too hard. Then the player should try to pass as many rounds as possible before that happens, [49]. 


\subsubsection{Helloquantum}

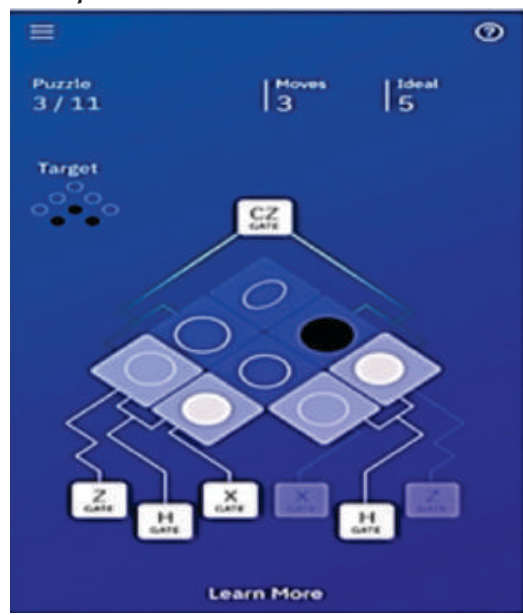

Figure 6. Quantum game interface "Hello quantum". Source: own.

Hello Quantum is a puzzle game designed to teach the introductory principles of quantum computing. This game was designed at IBM Research in Yorktown Heights, New York, in collaboration with Professor James Wootton of the University of Basel, Switzerland, [50].

\subsubsection{Meqanic}

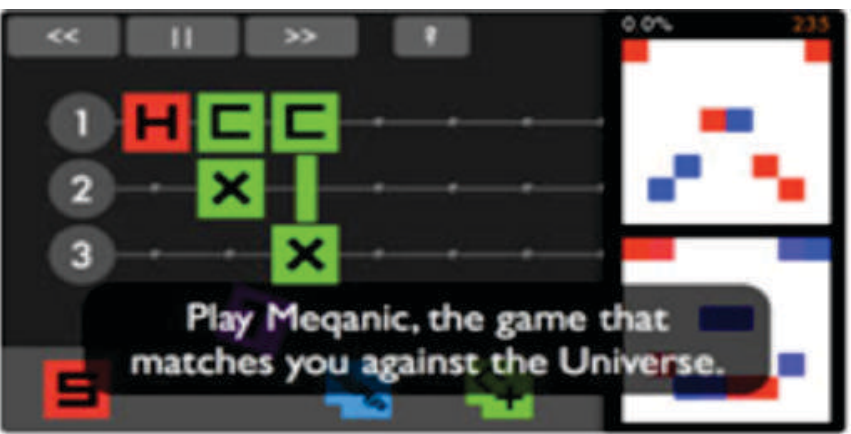

Figure 7. Quantum game interface "Meqanic". Source: own.

Meqanic is a physics-based puzzle game that confronts the user against the Universe. Meqanic is not evidenced as a game; it is a complete and precise quantum mechanics simulator. Meqanic has rules, but they are NOT intuitive because they are based on the quantum laws that govern the Universe itself. It's up to each player to solve them on the way. Being good at Meqanic requires a fundamental change in the way of thinking: it is a challenging game with high complexity and meaning, [51].

\subsubsection{Quantum game}

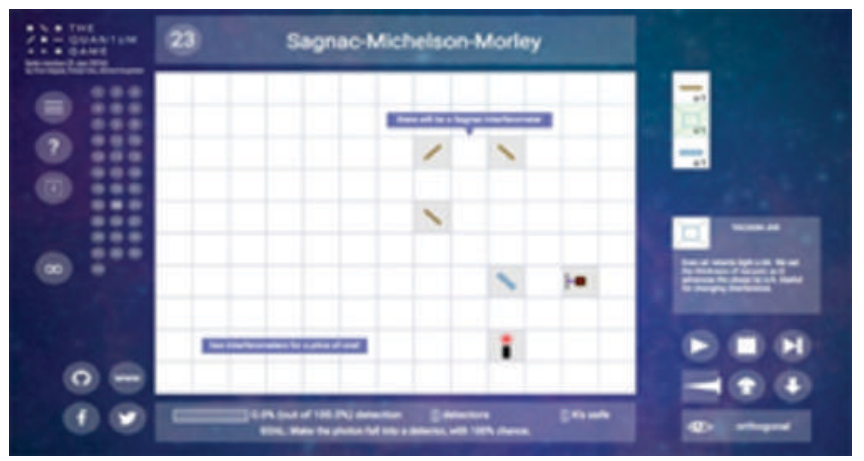

Figure 8. Quantum game interface "Quantum game". Source: own.

This is a game in which photons, mirrors, detectors, polarizing filters, and other elements interact on a table. Each level of the game presents a problem and all it requires is the correct placement of the components - knowing what each one of them does - to solve it and move to the next level. It is a kind of "quantum physics simulator", where each component behaves more or less as it would in the physical world. Many of the concepts and ideas of some of the levels of the game are used in quantum computing, [52].

\subsubsection{IBMQuantum computing}
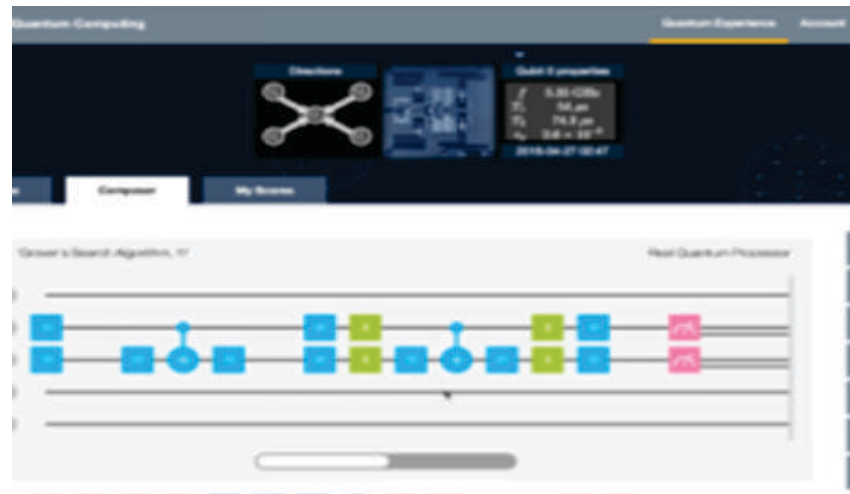

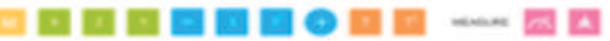

Figure 9. Quantum platform interface "IBM QUANTUM COMPUTING". Source: own.

Released in March 2017, IBM noted that $Q$ is an initiative to build universal, commercially available quantum computing systems for commercial and 
scientific applications. IBM $Q$ systems and services will be delivered through the IBM Cloud platform. IBM first opened public access to its quantum processors in 2017 to serve as an enabling tool for scientific research, a resource for university classrooms and a catalyst of excitement for the field, [53].

\subsection{Architecture of quantum computing platforms}

Today's physical quantum computing machinery (reminiscent of the first classical computers of the 1940s) is large in size. It requires a special physical environment and conditions to function properly. It is composed of five layers, Figure 10, three of which contain purely quantum hardware and circuits, and two of which consist of classical hardware and software:

\subsubsection{Quantum Layers, these layers can be thought} of as comprising the Quantum Processing Unit (QPU).

(a) Physical modules: includes quantum hardware that normally makes use of superconducting loops for the physical realization of Qubits. In addition, it also contains the physical qubit coupler / interconnection circuit among other elements that are necessary for Qubit control and addressing operations.

(b) Quantum logic gates - Physical circuits [16, x5.5] that make up quantum logic gates.

(c) Classic-quantum interface. Includes hardware and software that provides the interface between classical computers and a QPU.

\subsubsection{Classic layers}

(a) Quantum programming environment: provides elements such as: i) the quantum assembly language necessary to instruct a QPU, ii) the programming abstractions necessary to write quantum programs in a high-level programming language and iii) compatibility with simulators as well as IDEs, among others.

(b) Commercial applications: Quantum software applications written to meet commercial requirements, [54].

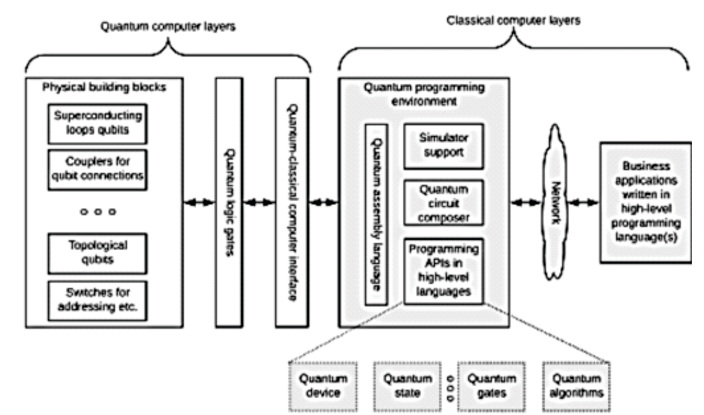

Figure 10. Quantum Computing Platform Architecture, [54].

\subsection{Quantic Programming Languages}

In the last decade several quantum programming languages were proposed: from imperative to functional; and from low to high level. Languages such as Quipper, ScaffCC / Scaffold, LIQUiji, QWire, Quil, $\mathrm{Q} \#$ and ProjectQ: allow the programming of large computers. Quipper is a strongly typed functional quantum programming language, integrated in Haskell; Scaffold is an independent programming language type $\mathrm{C}$ and its compiler ScaffCC takes advantage of the LLVM framework; QWire is integrated in the Coq test system; LIQUiji is embedded in $\mathrm{F} \#$; $\mathrm{Q} \#$ is an independent language type $\mathrm{F} \#$, and ProjectQ and Quil are integrated in Python. All of the above offer extensible frames for quantum circuit description and manipulation, and some offer gate decomposition and circuit optimization methods, some classical control flow, and the export of quantum circuits for representation or resource costs.

Theoretically, it would be enough if a quantum computing programming language supported the target hardware gate set. The similarity between such approach and the classical assembly language brought into existence quantum assembly languages such as QASM and OPEN-QASM. Although it is sufficient for today's quantum hardware, which is capable of performing some gate operations in less than 20 Qubits, programming in such language is neither scalable nor particularly easy to use. Instead, a quantum programming language should provide high-level abstractions to shorten development times and allow portability across a wide range of quantum hardware backends, similar to the current compilers for classical high-level languages such as $\mathrm{C}++$. Besides the purely classical and purely quantum subroutines, typical quantum algorithms also require that classical functions be evaluated in an input 
superposition, e.g. the modular exponentiation in Shor's algorithm for factorization.

\subsection{Trends, perspectives and challenges of the $Q C$}

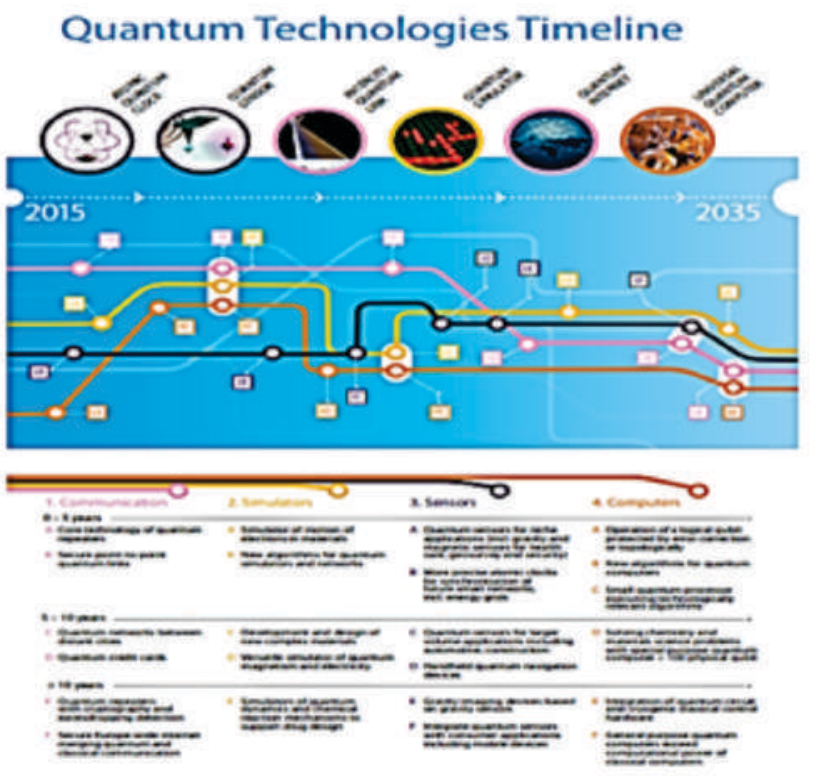

Figure 11. Quantum technologies time line $2015-$ 2035, [55].

Currently, the problem of creating a quantum computer is to maintain its stability over time; where each physicist adding a Qubit more implies that the engineering task becomes more laborious. Adding more Qubits makes the decoherence factor more vulnerable: where the state of the Qubit is degraded. Decoherence can be caused by interactions with the world, and problems are generated to maintain consistency in the system for more than several seconds. However, several advances have been made, in 1995 at the US National Institute of Standards and Technology the first quantum gate of two Qubits was built, and in 2005 a team led by Professor Rainer Blatt at the Institute of Experimental Physics in Austria built a quantum computer prototype using a few calcium ions. His group was at a tangled level of a maximum of eight calcium ions [9]. The National Institute of Standards and Technology now has a specific quantum computing program, like Yale University, which aims to offer real advances in this field that could revolutionize computing in the medium term. For the first time, two of these quantum processors have been linked together by superconducting circuits, creating a cavity through which quantum data is transmitted. To achieve the superconducting properties, the entire device was cooled to low temperatures. The importance of this discovery lies in the fact that this type of quantum bus will be used with high probability in future quantum computers or, at least, will be the basis of future communication buses. Now, the center of research will be the stability of communications with more than 6 Qubits, an initial reference that worked without apparent problems.

On the other hand, the greatest challenges that the quantum computer currently faces is the isolation of the Qubit, since any contact with its macroscopic world breaks the coherence, possible only with a slight observation, therefore: it is necessary to keep the Qubit totally isolated by retaining it with an ionic trap, that is: to keep it suspended between magnetic fields and laser beams: it is difficult to imagine what the quantum computer of the present and the future will look like, [55].

\section{Conclusions}

The state of the art was researched on the applications that have been created related to quantum computing, and how they help to improve the learning of this subject in the students. It has been evidenced, by a research incorporated in this document, that the free license tools are useful at the moment of learning. For the students of the District University Francisco José de Caldas, Technological Faculty, in Telematics Engineering, $80 \%$ of those surveyed stated that Quantum Game clarified definitions of quantum computing; likewise, when asked if the IBM platform and Quantum Game were complementary help for the subject of quantum computing: $65 \%$ said yes. It should be noted that $75 \%$ of the interviewees were facilitated in learning the Quantum Game tool.

On the other hand, it was possible to get to know the companies that research or contribute to science in terms of quantum computing; as well as the developments that have been generated, showing that the pioneering companies in this area are located in North America: IBM, Google and DWave Systems. Finally, it is concluded that quantum computing is a subject that companies and scientists intend to continue researching in order to surpass the 2000 Qubits computer of the company D-Wave, considered one of the most sophisticated computers at present.

Finally, in a time line between 2015 and 2035, it is likely that every household will have a quantum computer for daily use. In any case, the Qubit limit for quantum computers of this nature is not clear. 


\section{References}

[1] M. I. Dyakonov, "State of the Art and Prospects for Quantum Computing", 2013. https://doi.org/10.1002/9781118678107.ch20

[2] H. Vargas, "Alan Turing y la máquina de pensar", 2012. [Online]. Available at: https://www.proceso.com.mx/312974/31297 4-alan-turing-y-la-maquina-de-pensar

[3] A. Gramigna and J. C. G. Faraco, "Videojugando se aprende: renovar la teoría del conocimiento y la educación”, Revista científica de comunicación y educación, vol. 17, no. 33, 2009. https://doi.org/10.3916/c33-2009-03-007

[4] O. L. Palacio, L. Maldonado-Granados and L. Calderón-Villafáñez, "Guía para construir estados del arte”, 2016. [Online]. Available at: http://iconk.org/docs/guiaea.pdf

[5] S. Aaronson, "Quantum Computing since Democritus”, Cambridge University Press, $\begin{array}{lllll}2 & 0 & 1 & 3\end{array}$. https://doi.org/10.1017/CBO978051197930 $\underline{9}$

[6] P. Kaye, R. La amme and M. Mosca, "An Introduction to Quantum Computing", OUP Oxford, 2007.

[7] E. P. DeBenedictis, "A Future with Quantum Machine Learning", Computer, vol. 51, no. 2, 2018, pp. 68-71.

[https://doi.org/10.1109/MC.2018.1451646

[8] I. K. Sohn and J. Heo, "An Introduction to FaultTolerant Quantum Computation and its Overhead Reduction Schemes", Tenth International Conference on Ubiquitous and Future Networks (ICUFN), 2018. https://doi.org/10.1109/ICUFN.2018.84367 $\underline{51}$

[9] J. P. Hecht, "Fundamentos de computación cuántica”, 2005. [Online]. Available at: http://www.criptored.upm.es/guiateoria/gt $\underline{\text { m471a.htm }}$

[10] N. S. Yanofsky and M. A. Mannucci, "Quantum Computing for Computer Scientists", Cambridge University Press, 2008. https://doi.org/10.1017/CBO978051181388 $\underline{7}$

[11] P. Wittek, "Quantum Machine Learning", 2014, pp. 11-24. https://doi.org/10.1016/B978-012-800953-6.00002-5

[12] Gale, "Academic OneFile", 2018. [Online]. Ava il a ble:

https://www.gale.com/intl/c/academiconefile

[13] T. Monz, D. Nigg, E. A. Martinez, M. F. Brandl, P. Schindler, R. Rines, S. X. Wang, I. L. Chuang and R. Blatt, "Realization of a scalable Shor algorithm “, Science, vol. 351, no. 6277, 2016, pp. $1068-1070$. . https://doi.org/10.1126/science.aad9480

[14] M. Raisinghani, "Quantum computers: A new paradigm in information technology", Informing Science, vol. 4, no. 1, 200 1, pp. 11-16.

[15] G. P. Mango, "Computación Cuántica”, 2013. [ O n line ]. Avail a ble a t: https://edoc.pub/computacion-cuantica-19pdf-free.html

[16] Studylib, "Computadora cuántica”, 2016. [ O n l ine e. Avail a ble a t: http://studylib.es/doc/783060/computadoracuantica-en-1985-david-deutsch-dio-una-base.

[17] L. R. Camargo and L. C. Osorio-Ovalle, "Computación Cuántica", 2011. [Online]. Available at: https://edoc.site/computacioncuantica-monografia-pdf-free.html

[18] S. Muñoz, "Los físicos descubren cómo teletransportar energía”, 2014. [Online]. Available at:

http://www.cosmonoticias.org/los-fisicos- 
descubren-como-teletransportar-energia/

[19] A. D. Caro and J. Samborski-Forlese, "Brevísima Introducción a la Computación Cuántica", 2006 . [O n line ]. Available at: http://software.imdea.org/ julian/docs/Sam borski.Forlese.2006.JAI.pdf

[20] EcuRed, "Algoritmo de Grover", 2017. [O n l ine ]. A vail a ble a t : https://www.ecured.cu/Algoritmo_de_Grover

[21] Wikipedia, "Computación cuántica", 2018. [ O n line ]. Avail a ble a t: https://es.wikipedia.org/wiki/Computaci\%C 3\%B3n_cu\%C3\%A1ntica

[22] El Mundo, "IBM presenta su primer ordenador cuántico, un nuevo 'mesías' para el mundo de la informática”, 2017. [Online]. Available at: http://www.elmundo.es/tecnologia/2017/03 /06/58bd3af5268e3ef45d8b4632.html

[23] Timetoast, "Avances en computación cuántica", 2013 , [Online]. Available at: https://www.timetoast.com/timelines/avance s-en-computacion-cuantica

[24] W. Guzmán, "Historia de la informática del 2005 al 2012", 2013. [Online]. Available at: https://www.timetoast.com/timelines/histori a-de-la-informatica-del-2005-al-2012--16

[25] ScienceDaily, "12 Qubits alcanzados en la búsqueda de información cuántica”, 2006. [ $\mathrm{O} n \mathrm{line}$. A v a i l a ble a t: https://www.sciencedaily.com/releases/2006 /05/060508164700.htm

[26] Studylib, "La computación cuántica", 2015. [ O n l i n e ]. A va i l a ble a t : http://studylib.es/doc/711084/lacomputaci\%C3\%B3n-cu\%C3\%A1ntica

[27] J. M. Patiño-Gutiérrez, "Arquitectura de las computadoras y la computación cuántica", 2013 . [Online]. Available at: https://www.researchgate.net/publication/26 2871304 Arquitectura de las computadoras _y la_computacion_cuantica

[28] Infobae, "El asalto de la computación cuántica, relatada por uno de sus principales protagonistas", 2014. [Online]. Available at: https://www.infobae.com/2014/08/06/1585 797-el-asalto-la-computacion-cuantica- relatada-uno-sus-principales-protagonistas/

[29] M. Lynley, "La primera computadora cuántica comercial del mundo vendida a Lockheed Martin, 2011. [Online]. Available at: https://venturebeat.com/2011/05/27/firstquantum-computer-sold/

[30] H. Knowles, "Researchers seek to advance quantum computing", 2017. [Online]. Available at:

https://www.stanforddaily.com/2017/05/12/ researchers-seek-to-advance-quantumcomputing/

[31] A. Wichert, "Principles of Quantum Arti cial Intelligence”, World Scientific Pub Co Inc, 2014.

[32] C. H. Bennett and D. P. DiVincenzo, "Información y computación cuántica", [O n l in e ]. Ava il a ble a t: http://faeuato.us.es/Adan/Carpetas/Publicati ons/To02\%20BDoo.pdf

[33] B. R. Cifuentes-Pérez, "Computación Cuántica", thesis, Universidad de San Carlos de Guatemala, Guatemala, 2006.

[34] J. M. Renes, "The Physics of Quantum Information Complementarity, Uncertainty, and Entanglement", International Journal of Quantum Information, vol. 11, no. 08, 2013. https://doi.org/10.1142/S02 1974991330002 $\underline{7}$

[35] V. M. Bonillo, "Principios Fundamentales de la Computación cuántica”, 2013. [Online ]. Av a i l a ble a t: http://www.lidiagroup.org/images/descargas /varios/011_ccuantica.pdf

[36] IBM, "IBM Innovation Exchange 2018”, 2018. [ O n l ine $]$. Ava il a ble a t : https://www.ibm.com/co-es/

[37] D-Wave, "Computación cuántica para el mundo real hoy", 2017. [Online]. Available at: https://www.dwavesys.com/quantumcomputing

[38] C. Q. Choi, “Qubit Revolution”, 2017. [Online]. Available at: http://www.aseeprism.org/qubit-revolution/ 
[39] T. Simonite, "Una diminuta 'start-up' quiere vencer a Google con su primer chip cuántico”, 2016 . [Online $]$. Available at: https://www.technologyreview.es/s/5549/un a-diminuta-start-quiere-vencer-google-consu-primer-chip-cuantico

[40] Gestión, "IBM sube la presión sobre sus rivales con computadora cuántica”, 2017. [Online]. A v a i 1 a b l e a $\mathrm{t}$ : https://gestion.pe/economia/empresas/ibmsube-presion-rivales-computadora-cuantica$\underline{150176}$

[41] IBM, "La computación cuántica: La realidad que revolucionará el mundo empresarial”, 2017. [ $\mathrm{O}$ n l ine $]$. Ava il a ble a t : https://www.ibm.com/blogs/think/eses/2017/07/27/Qubits-un-nuevo-mundoempresarial/

[42] S. Angulo, "Google es el nuevo rey de la computación cuántica”, 2018. [Online]. Available at: http://www.enter.co/chipsbits/computadores/google-nuevo-reycomputacion-cuantica/

[43] Microsoft, "Con el simulador de cómputo cuántico, Microsoft ofrece un vistazo al futuro del cómputo", 2015. [Online]. Available at: https://news.microsoft.com/es-xl/con-elsimulador-de-computo-cuantico-microsoftofrece-un-vistazo-al-futuro-del-computo/

[44] Computerworld, "D-Wave lanza un ordenador cuántico de 2.000 Qubits”, 2016. [Online]. Available at:

http://www.computerworld.es/innovacion/d wave-lanza-un-ordenador-cuantico-de-2000Qubits

[45] IEEE, "La organización profesional técnica más grande del mundo para el avance de la tecnología”, 2011. [Online]. Available at: https://nodoka.co/es/noticias/institute-ofelectrical-and-electronics-engineers-ieee

[46] Electrónicos online, "IEEE prepara el camino para estandarizar la computación cuántica”, 2017 . [Online]. Available at: https://www.electronicosonline.com/ieeeprepara-el-camino-para-estandarizar-lacomputacion-cuantica/
J. Wootton, "Presentamos el primer juego del mundo para una computadora cuántica”, 2017. [O n l ine ]. Ava i l a ble a t: https://medium.com/@decodoku/introducin g-the-worlds-first-game-for-a-quantumcomputer-50640e3c22e4

[48] Technology Review, "Ayude a revolucionar la computación cuántica con este juego de puzles en 3D", 2016. [Online]. Available at: http://www2.technologyreview.es/informatic a/51957/ayude-a-revolucionar-lacomputacion-cuantica-con/

[49] J. Wootton, "Usando un simple juego de rompecabezas para comparar computadoras cuánticas”, 2018. [Online]. Available at: https://medium.com/@decodoku/understan ding-quantum-computers-through-a-simplepuzzle-game-a290dde89fb2

[50] Hello Quantum, "Acerca de Hello Quantum", 2016 . [Online]. Available at: https://helloquantum.mybluemix.net/\#about

[51] Apprecs, "Meqanic", 2017, [Online]. Available a t :

https://apprecs.com/ios/818589077/meqanic

[52] Microsiervos, "The Quantum Game, o cómo aprender computación cuántica jugando con rompecabezas lógicos", 2017. [Online]. Available at:

https://www.microsiervos.com/archivo/jueg os-y-diversion/the-quantum-game.html

[53] Gale, "Fine Arts and Music Collection", 2017. [ O n l ine ]. A va i l a ble a t : https://www.gale.com/intl/c/fine-arts-andmusic-collection

[54] B. Sodhi, "Quality Attributes on Quantum Computing Platforms", 2018. [Online]. Available at:

https://arxiv.org/pdf/1803.07407.pdf

[55] J. P. Rúa Vargas and J. W. Branch, "Estado del arte de la computación cuántica”, 2009. [ $\mathrm{O} n \mathrm{line}$ ]. A vail a ble a t: http://bdigital.unal.edu.co/23468/1/2037968840-1-PB.pdf 